\title{
Eczema and Risk of Cardiovascular Disease: Heightened Awareness Needed
}

\author{
Akira Fujiyoshi
}

Department of Hygiene, Wakayama Medical University, Wakayama, Japan

\section{See article vol. 26: 775-782}

Recent evidence suggests an association of chronic inflammatory skin diseases, such as psoriasis and atopic dermatitis, with the risk of cardiovascular diseases (CVD). Eczema and dermatitis are frequently used interchangeably, and chronic eczema (chronic dermatitis) is a common skin disorder worldwide ${ }^{1)}$. Among the subtypes of chronic eczema, the association of psoriasis with CVD has been reported to be strong ${ }^{2)}$. However, the association of atopic dermatitis (atopic eczema) with CVD remains unestablished ${ }^{2)}$.

Atopic dermatitis is the most common type of eczema $^{3)}$ and traditionally considered a disease that occurs during childhood; however, approximately $50 \%$ of these childhood cases can extend into adulthood, evolving into a life-long condition ${ }^{4)}$. The estimated prevalence of adults with eczema or atopic dermatitis is $6.9 \%$ and $10.2 \%$ in Japan ${ }^{5)}$ and the United States ${ }^{6}$, respectively. Given such a high prevalence, even a small increase in the risk of CVD would be important from a public health perspective ${ }^{7)}$. Nonetheless, only a limited number of population-based studies have examined a longitudinal association of chronic dermatitis with CVD in adults. For example, a recent meta-analysis on population-based studies found that only eight published papers were longitudinal. The results of those eight studies were somehow mixed, and most of them sampled from Western countries in which the structure of CVD substantially differs from that in East Asia, including Japan ${ }^{8)}$.

Given the relative paucity of pertinent studies, the population-based epidemiological study by Nishida et al. ${ }^{9)}$ provides additional evidence that supports an association of chronic eczema with coronary heart disease (CHD) in a Japanese population. After excluding participants with any previous history of CHD, stroke or cancer at baseline (1988 to 1990 , the entire cohort was initially recruited from 45 areas across Japan), the authors followed up more than 85,000 Japanese men and women for approximately 20 years. During the follow-up, they observed 1,174 deaths from CHD, 979 from heart failure, 366 from cardiac arrhythmia, and 2454 from total stroke. Individuals who reported having eczema "often" at baseline had a 39\% (95\% confidence interval, 12\%-72\%) higher risk of deaths due to CHD than those who reported less frequent eczema in an age- and sexadjusted model. This positive association was slightly attenuated but persisted even with further adjustment for cardiovascular risk factors, including body mass index, history of hypertension, diabetes, perceived mental stress, educational attainment, alcohol intake, smoking status, and other lifestyle factors. Further, the risk of death due to CHD was higher by 30\% (95\% confidence interval, 5\%-61\%) in a multivariable adjusted model. However, no association of frequent eczema with other types of cardiovascular diseases, such as stroke, was observed. The authors speculated that the reason for this lack of association is the smaller contribution of inflammation in the pathogenesis of stroke relative to CHD and smaller numbers of events observed for other cardiovascular diseases. Of note, the significant positive association with CHD was maintained with further adjustment for serum cholesterol level in a subsample of participants who had information on serum cholesterol concentration $(n=26,222)$. To my knowledge, this is the first population-based longitudinal study that showed a positive association of frequent eczema with death due to CHD in Japanese men and women.

Strengths of their work include a large sample size with a nation-wide recruitment, long follow-up period, and reasonably extensive adjustment for potential confounders. One limitation of the work is its non-specific nature of exposure assessment of eczema. However, given that atopic dermatitis is the

Address for correspondence: Akira Fujiyoshi, Department of Hygiene, Wakayama Medical University, 811-1 Kimiidera, Wakayama, 641-8509, Japan

Email: afujiy@wakayama-med.ac.jp

Received: February 26, 2019 Accepted for publication: March 6, 2019

Copyright@2019 Japan Atherosclerosis Society

This article is distributed under the terms of the latest version of CC BY-NC-SA defined by the Creative Commons Attribution License. 
most common type of eczema, this limitation may not be crucial. Another limitation includes the lack of information on treatment status for eczema. Also, a question remains as to whether self-reported frequency was an appropriate surrogate for disease severity or activity. The aforementioned meta-analysis concluded that increasing severity of atopic dermatitis was associated with a wide range of CVD outcomes, including angina, heart failure, myocardial infarction, and cardiovascular death ${ }^{2)}$.

Speculated mechanisms linking chronic dermatitis with CVD include inflammation, which is in part related to impaired skin barrier, platelet dysfunction, and decreased fibrinolysis - all of which can contribute to increased clotting - and treatment for eczema ${ }^{2)}$. In fact, observational evidence suggests that severe eczema is more clearly associated with an increased risk of $\mathrm{CVD}^{2,10)}$, and long-term treatment for chronic eczema by itself appears to increase the risk ${ }^{11)}$. Therefore, future studies are encouraged to include treatment status for eczema in their analyses. Another focus for future research is to elucidate potential mechanism(s) linking eczema with CVD. For example, some, but not all, pertinent studies reported an increased prevalence of cardiovascular risk factors such as hypertension, hyperlipidemia, obesity, and sleep disorders among individuals with eczema ${ }^{4)}$ (similar findings were reported in Nishida's work $)^{9)}$. These factors may be an important set of mediators linking eczema with the risk of CVD, although the reported associations were statistically independent of such factors according to studies by Nishida and by others.

In summary, in their longitudinal populationbased study, Nishida et al. showed a positive and independent association of frequent eczema with the risk of death due to CHD in a sample of Japanese men and women. Further study is needed to elucidate its mechanism and to clarify any association with other cardiovascular diseases. Until further clarified, clinicians and researchers should heighten their awareness of the link, making preventive efforts of cardiovascular disease by early treatment and better control of chronic eczema, particularly atopic dermatitis and psoriasis.

\section{Conflicts of Interest}

None.

\section{References}

1) Williams H, Stewart A, von Mutius E, Cookson W, Anderson HR, International Study of A, Allergies in Childhood Phase O and Three Study G: Is eczema really on the increase worldwide? J Allergy Clin Immunol, 2008; 121: 947-954 e915

2) Ascott A, Mulick A, Yu AM, Prieto-Merino D, Schmidt M, Abuabara K, Smeeth L, Roberts A and Langan SM: Atopic eczema and major cardiovascular outcomes: A systematic review and meta-analysis of population-based studies. J Allergy Clin Immunol, 2019; 143: 1821-1829

3) Weidinger $S$ and Novak N: Atopic dermatitis. Lancet, 2016; 387: 1109-1122

4) Arima K, Gupta S, Gadkari A, Hiragun T, Kono T, Katayama I, Demiya $S$ and Eckert L: Burden of atopic dermatitis in Japanese adults: Analysis of data from the 2013 National Health and Wellness Survey. J Dermatol, 2018; 45: 390-396

5) Saeki H, Tsunemi Y, Fujita H, Kagami S, Sasaki K, Ohmatsu H, Watanabe A and Tamaki K: Prevalence of atopic dermatitis determined by clinical examination in Japanese adults. J Dermatol, 2006; 33: 817-819

6) Silverberg JI and Hanifin JM: Adult eczema prevalence and associations with asthma and other health and demographic factors: a US population-based study. J Allergy Clin Immunol, 2013; 132: 1132-1138

7) Ingram JR: Atopic eczema and cardiovascular disease. BMJ, 2018; 361: k2064

8) Ueshima H: Explanation for the Japanese paradox: prevention of increase in coronary heart disease and reduction in stroke. J Atheroscler Thromb, 2007; 14: 278-286

9) Nishida Y, Kubota Y, Iso H, Tamakoshi A and Group JS: Self-Reported Eczema in Relation with Mortality from Cardiovascular Disease in Japanese: the Japan Collaborative Cohort Study. J Atheroscler Thromb, 2019; 26: 775 782

10) Silverwood RJ, Forbes HJ, Abuabara K, Ascott A, Schmidt M, Schmidt SAJ, Smeeth L and Langan SM: Severe and predominantly active atopic eczema in adulthood and long term risk of cardiovascular disease: population based cohort study. BMJ, 2018; 361: k1786

11) Wei L, MacDonald TM and Walker BR: Taking glucocorticoids by prescription is associated with subsequent cardiovascular disease. Ann Intern Med, 2004; 141: 764-770 